\title{
Multiple bilateral forearm nodules
}

\section{Robert W. Morris ${ }^{1}$ (D) $\cdot$ Youssef Al Hmada ${ }^{2} \cdot$ Joseph R. Chaudry ${ }^{1} \cdot$ Kathryn M. Nutter $^{1} \cdot$ Chris D. Reed $^{1}$}

Published online: 3 August 2019

(C) ISS 2019

44 year-old female with several month history of firm, painful nodules involving the bilateral forearms. MRI with contrast of the left (Fig. 1) and right (Fig. 2) forearms was performed. Excision of a painful right forearm nodule was also performed (Fig. 3).

The diagnosis can be found at https://doi.org/10.1007/s00256-01903296-9

$\triangle$ Robert W. Morris

rwmorris@umc.edu

1 Department of Radiology, University of Mississippi Medical Center, 2500 North State Street, Jackson, MS 39216, USA

2 Department of Pathology and Laboratory Medicine, University of Mississippi Medical Center, 2500 North State Street,

Jackson, MS 39216, USA 


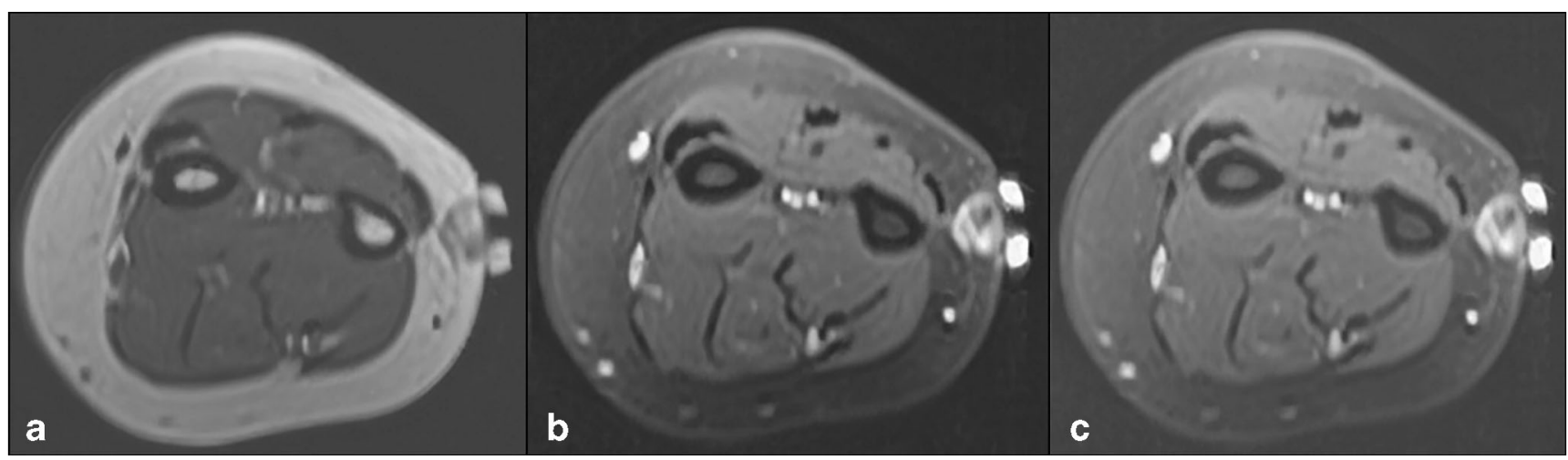

Fig. 1 a Axial TI, b axial PD fat sat and $\mathbf{c}$ axial T1 fat sat with contrast of the left forearm

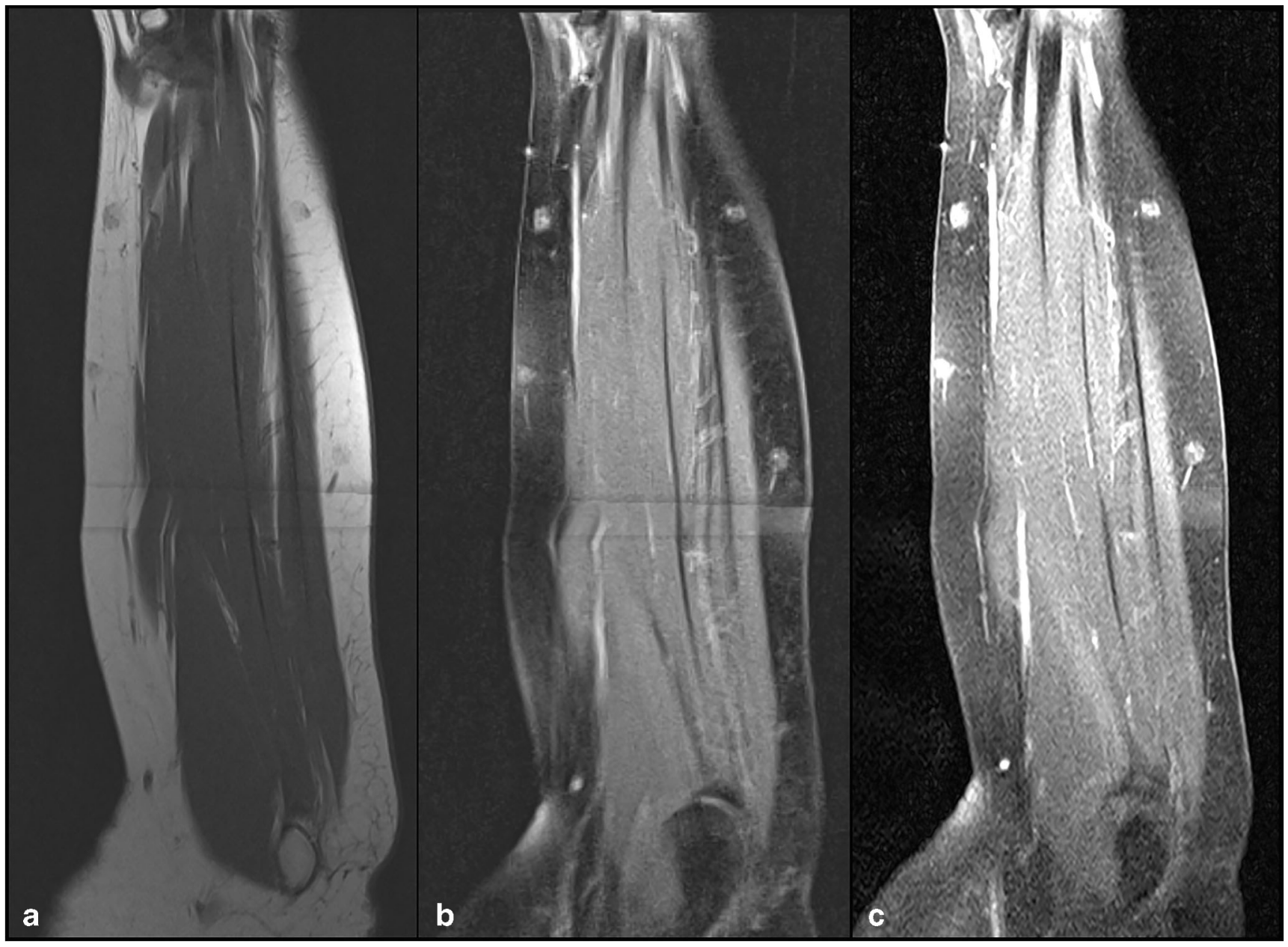

Fig. 2 a Coronal TI, b coronal PD fat sat and $\mathbf{c}$ coronal T1 fat sat with contrast of the right forearm 


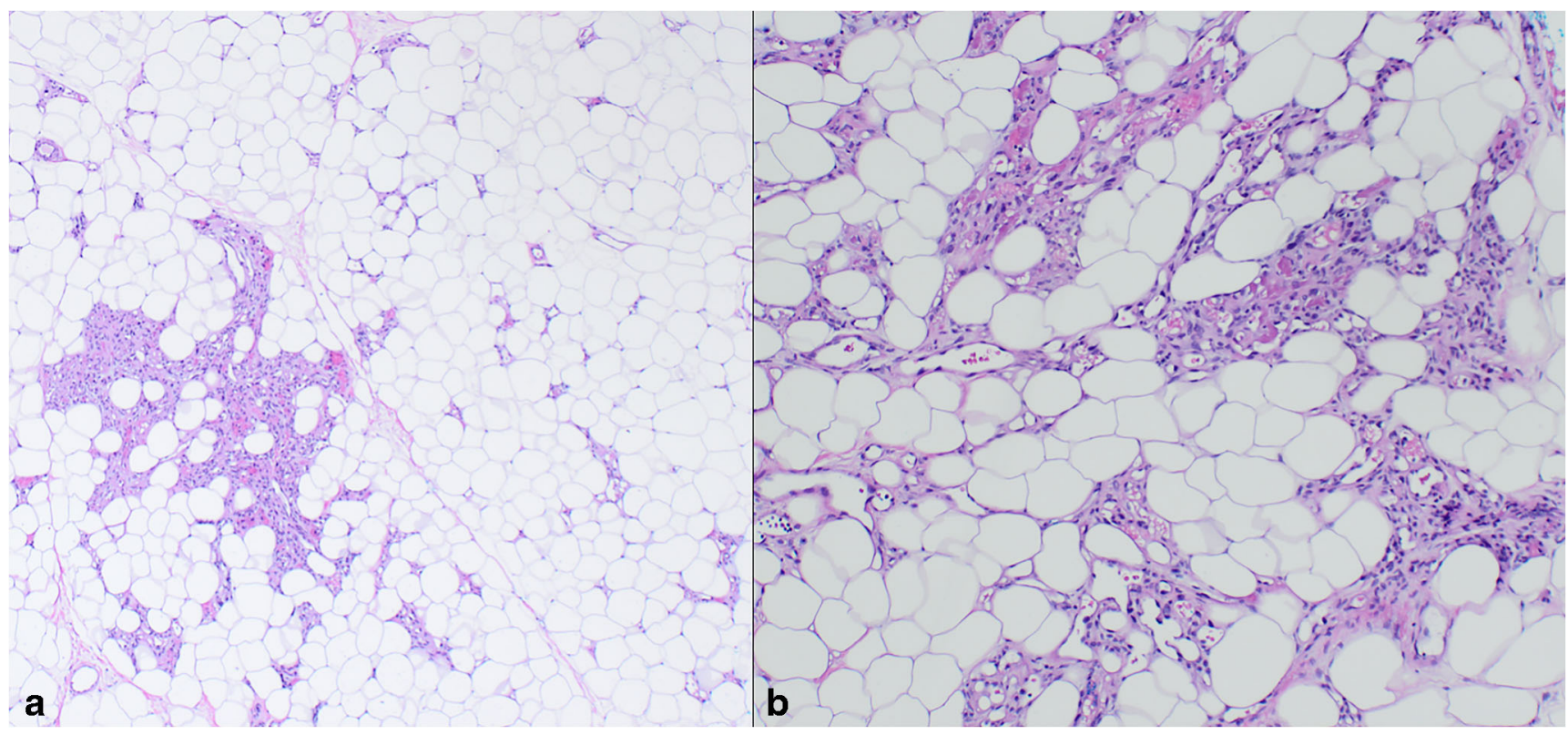

Fig. 3 Hematoxylin and eosin stained sections of the excised right forearm nodule at a 40x and $\mathbf{b} 100 \mathrm{x}$ magnification

Publisher's note Springer Nature remains neutral with regard to jurisdictional claims in published maps and institutional affiliations. 